\title{
Enhancement of spin-dependent hole delocalization in degenerate asymmetric double quantum wells
}

\author{
M. Kemerink and P. M. Koenraad \\ COBRA Interuniversity Research Institute, Eindhoven University of Technology, P.O. Box 513, 5600 MB Eindhoven, The Netherlands \\ P. C. M. Christianen, A. K. Geim, and J. C. Maan \\ High-Field Magnet Laboratory, University of Nijmegen, Toernooiveld, 6525 ED Nijmegen, The Netherlands \\ J. H. Wolter \\ COBRA Interuniversity Research Institute, Eindhoven University of Technology, P.O. Box 513, 5600 MB Eindhoven, The Netherlands \\ M. Henini \\ Department of Physics, University of Nottingham, Nottingham NG7 2RD, United Kingdom
}

(Received 3 October 1995; revised manuscript received 26 December 1995)

\begin{abstract}
Exact, self-consistently calculated eigenfunctions and eigenvalues of the valence band in degenerate asymmetric double quantum wells are obtained from the full $4 \times 4$ Luttinger Hamiltonian for different hole densities. We found the solutions to be extremely sensitive to the charge density. The charge induces an extra asymmetry in the confining potential and leads to a smaller separation of the hole levels in the adjacent wells. This strongly enhances the different leakage of wave functions with opposite magnetic moment, called spin-dependent hole delocalization (SDHD). Furthermore, it is shown that the SDHD of the heavy-hole states can be enhanced by increasing the confinement of the light-hole states through adjustment of the height of the confining barriers. The theoretical results are shown to be in excellent agreement with transport and optical experiments, which are proven to be largely determined by space-charge effects.
\end{abstract}

PACS number(s): 73.20.Dx, 73.40.Kp, 78.66.Fd

\section{INTRODUCTION}

In the past decades the energy bands of low-dimensional semiconductor structures have been intensively studied, both by experimental and theoretical means. For the $J=1 / 2$ electron states, originating from $S$-type molecular orbitals, an almost parabolic behavior was found. Only for large wave numbers and high magnetic fields are the deviations from parabolicity significant. The valence band, in contrast, is strongly non-parabolic. Since the hole states are formed from $P$-type molecular orbitals $(L=1)$, the spin-orbit interaction becomes important and splits the hole states in a split-off duplet with $J=1 / 2$ and a heavy-hole-light-hole quadruplet with $J=3 / 2$. The interactions between these bands result in strongly nonparabolic dispersion relations. Due to this complexity, it has only recently been recognized that structures can be developed that take advantage of the physical properties of the valence band, both to explore new physics and to improve device performance. ${ }^{1}$

One of the aforementioned structures that has been intensively studied in the past few years is the asymmetric double quantum well (ADQW), ${ }^{2-8}$ consisting of two (single) QW's of different width, separated by a tunnel barrier; see Fig. 1 . As a device application, such structures can, e.g., be used as bias-voltage tunable infrared detectors ${ }^{2,3}$ or as velocity modulation transistors. ${ }^{4}$ More fundamentally, ADQW's can show strong tunneling-dependent level broadening when the scattering intensities in the left and right wells are different. ${ }^{5}$ One of the most interesting features is the different leakage of hole wave functions of different magnetic moment, leading to a macroscopic separation of "spin-up" and "spin- down" states. This effect was coined "spin-dependent hole delocalization" (SDHD) by Goldoni and Fasolino. ${ }^{6,7}$ Due to the asymmetry of the potential, the Kramers degeneracy of the hole levels is lifted, leading to spin- $\left(m_{J}= \pm 3 / 2, \pm 1 / 2\right)$ dependent hole delocalization, even when there is no resonance between levels in the left and right well.

In most theoretical work ${ }^{5-8}$ the effects of band filling on the hole states are not taken into account. These are essential to explain most experimental results quantitatively and as we point out, even qualitatively. In this paper we will show the extreme sensitivity of the hole energy levels and optical oscillator strengths on the presence of a degenerate hole gas. Furthermore, we will show that the SDHD is strongly enhanced when the band bending resulting from the presence of holes is included.

This paper will be organized as follows. In Sec. II the theoretical framework in which the numerically exact solutions of both the Schrödinger and Poisson equations are obtained will be presented. In Sec. III numerical results will be presented, which will be compared with experiments in Sec. IV. A summary will be given in Sec. V.

\section{THEORY}

In this section we will outline our approach to solve the Luttinger Hamiltonian. We have used a commercially available routine to obtain exact eigenvalues and eigenfunctions from a set of coupled first-order differential equations. The routine used solves a two-point boundary-value problem, described by a set of coupled first-order differential equations, using a deferred correction technique and Newton iteration. ${ }^{9}$ 
Since this method is not limited by a (finite) basis set of functions to expand the eigenstates, it is numerically exact, i.e., within a user-specified tolerance, typically $10^{-5}$. This is similar to expanding the eigenfunctions in an infinite basis set.

In most theoretical studies ${ }^{10-14}$ on valence bands that appeared in the past decades, the hole eigenstates are expanded in a limited set of basis functions and are calculated by matrix diagonalization. This method is known to give rise to significant deviations for larger wave numbers, unless the number of basis functions is drastically increased. ${ }^{10}$ Other, exact, methods ${ }^{15-17}$ are applicable only to highly symmetric structures, such as empty single quantum wells. The method presented here is suited for any given potential, under the limitation that a good starting solution can be generated. Also the effects of strain or a magnetic field can easily be included, ${ }^{18}$ and easy access to wave functions and their derivatives is provided. However, in this paper we will be concerned only with the asymmetric double quantum well in zero magnetic field. To calculate the hole energy levels we used the Luttinger Hamiltonian, ${ }^{19}$ with inclusion of warping, in the spin-orbit basis with $J_{z}=(3 / 2,-1 / 2,1 / 2,-3 / 2)$. The confining potential is assumed to be parallel to the $z$ axes and hole energy is counted positive. The split-off band components of the lowest hole levels of the $J=3 / 2$ multiplet are known to be negligible, ${ }^{15}$ which reduces the Luttinger Hamiltonian to $4 \times 4$. Furthermore, we will neglect linear $k$ terms, arising from the lack of inversion symmetry of the GaAs crystal, since they only give rise to small energy splittings 11,20

$$
\begin{array}{r}
\frac{\hbar^{2}}{2 m_{0}}\left[\begin{array}{cccc}
H_{h}+V(z) & c & -b & 0 \\
c^{+} & H_{1}+V(z) & 0 & b \\
-b^{+} & 0 & H_{1}+V(z) & c \\
0 & b^{+} & c^{+} & H_{h}+V(z)
\end{array}\right] \\
\times\left[\begin{array}{c}
\psi_{1}(z) U_{1} \\
\psi_{2}(z) U_{2} \\
\psi_{3}(z) U_{3} \\
\psi_{4}(z) U_{4}
\end{array}\right],
\end{array}
$$

where

$$
\begin{gathered}
H_{h}=\left(k_{x}^{2}+k_{y}^{2}\right)\left(\gamma_{1}+\gamma_{2}\right)+k_{z}^{2}\left(\gamma_{1}-2 \gamma_{2}\right), \\
H_{1}=\left(k_{x}^{2}+k_{y}^{2}\right)\left(\gamma_{1}-\gamma_{2}\right)+k_{z}^{2}\left(\gamma_{1}+2 \gamma_{2}\right), \\
b=\sqrt{3} \gamma_{3}\left(k_{x}-i k_{y}\right) k_{z}, \\
c=-\sqrt{3}\left[\gamma_{2}\left(k_{x}^{2}-k_{y}^{2}\right)-2 i \gamma_{3} k_{x} k_{y}\right], \\
k_{z}=\frac{1}{i} \frac{\partial}{\partial z} .
\end{gathered}
$$

The $U_{i}$ are the periodic Bloch functions in the basis $m_{J}=\{3 / 2,-1 / 2,1 / 2,-3 / 2\}$. In order to reduce the numerical effort we have applied the Broido-Sham transformation, ${ }^{11,21}$ making (1) block diagonal. The remaining set of coupled second-order differential equations is then transformed into first order, giving two sets of six equations, when equations
TABLE I. Energies of the first two heavy, light, and electron levels in a filled or empty ADQW (59.4- and 42.4- $\AA$ wells, 33.9- $\AA$ barrier) with $40 \% \mathrm{Al}$ barriers for two different interface conditions. See the text for further explanation.

\begin{tabular}{lcccc}
\hline \hline & \multicolumn{2}{c}{ Empty structure } & \multicolumn{2}{c}{$p=1.5 \times 10^{16} \mathrm{~m}^{2}$} \\
Level & $\frac{1}{m^{*}} \frac{\partial \Psi}{\partial z}$ & $\frac{\partial \Psi}{\partial z}$ & $\frac{1}{m^{*}} \frac{\partial \Psi}{\partial z}$ & $\frac{\partial \Psi}{\partial z}$ \\
continuous & continuous & continuous & continuous \\
\hline HH0 & 16.62 & 16.86 & 10.69 & 10.95 \\
HH1 & 27.10 & 27.58 & 12.62 & 12.56 \\
LH0 & 39.97 & 41.84 & 32.38 & 34.22 \\
LH1 & 63.81 & 68.37 & 48.32 & 52.52 \\
E0 & 60.89 & 65.93 & 66.76 & 71.74 \\
$E 1$ & 93.55 & 104.21 & 107.81 & 118.49 \\
\hline \hline
\end{tabular}

for normalization and energy continuity are included. Standard boundary conditions are applied at the outer boundaries of the calculation interval.

The choice between flux conservation or wave-functioncontinuity imposing interface conditions (IC's), i.e., keeping $\left(1 / m^{*}\right)(d \Psi / d z)$ or $(d \Psi / d z)$ continuous at the interfaces, ${ }^{22,23}$ can be made by simply taking the Luttinger parameters of the barrier material $\left(\mathrm{Al}_{x} \mathrm{Ga}_{1-x} \mathrm{As}\right)$ different or the same as those of the well material ( $\mathrm{GaAs}$ ), respectively. However, when flux-conserving IC's are chosen, the Luttinger parameters $\gamma_{i}$ become $z$ dependent and no longer commute with the operators $k_{z}$ and $k_{z}^{2}$. Therefore expressions of the type $\gamma_{i} k_{z}$ and $\gamma_{i} k_{z}^{2}$ have to be replaced by their respective anticommutators. ${ }^{24}$ Since we will assume low Al content $(25 \%)$ barriers and focus on the lowest subbands, which penetrate only a few atomic layers into the barriers, the difference between the two will be small and will generally fall below experimental resolution. For the standard structure of Sec. III we have listed the first heavy, light, and electron levels in Table I for both interface conditions. In the remainder of this paper we have applied the wave functioncontinuity imposing IC's, unless stated otherwise. The parameters used in the calculations are $\gamma_{1}=6.85, \gamma_{2}=2.1$, and $\gamma_{3}=2.9$ for GaAs and $\gamma_{1}=3.45, \gamma_{2}=0.68$, and $\gamma_{3}=1.29$ for AlAs. ${ }^{25}$ Intermediate values are calculated using linear interpolation.

The actual calculation is split in two parts: a part for $k=0$ in which self-consistency is obtained and one for finite $k$. The $k=0$ calculation starts with solving (1) for a flatband potential, taking advantage of the fact that (1) decouples into four Schrödinger-type equations, which are easily solvable. The starting solutions for this procedure are generated as even and odd combinations of half or whole-period sinuses, localized entirely in the separate wells. For the lowest subbands (a half period sinus in each well) the even combinations correspond to the lowest energy; see Fig. 1.

The wave functions obtained in this way are used to calculate the charge distribution $\rho(z)$ in the system. Poisson's equation is then solved by numerical integration of $\rho(z)$ and the charged acceptor distribution

$$
\frac{\partial^{2}}{\partial z^{2}} V_{c}(z)=\frac{e}{\epsilon_{0} \epsilon_{r}} \rho(z) .
$$


The charge present in the wells is distributed over the $k=0$ wave functions assuming parabolic bands with effective masses given by $m_{0} /\left(\gamma_{1}+\gamma_{2}\right)$ and $m_{0} /\left(\gamma_{1}-\gamma_{2}\right)$ for heavy and light bound bands, respectively, where $m_{0}$ is the electron rest mass. The doping is assumed to be distributed over two $\delta$ layers on the left- and right-hand sides of the structure, in such a way that no net voltage drop is present over the structure. In practice this means that the doping is almost equally distributed. A weighted average of the new and old potentials is then used to calculate the next iteration. This procedure is iterated until the potential is stable within a predefined limit $\left(10^{-5}\right)$. The final potential is then used for the calculation of all hole levels at finite $k$ from (1).

For highly degenerate systems the parabolic approximation for charge distribution over the $k=0$ wave functions, implicitly assuming that the wave functions are independent of $k$, will induce an error in $\rho(z)$, but this effect was checked to be small. The lowest hole levels (HH0,1 and LH0,1) calculated exactly were within $0.5 \mathrm{meV}$ of those calculated in the parabolic approximation of $\rho(z)$, for densities up to $1.5 \times 10^{12} \mathrm{~cm}^{-2}$. Because of the enormous difference in computational effort, we will use the latter method.

\section{NUMERICAL RESULTS}

There are three relevant dimensions in any ADQW (the width of both wells and their separating barrier) that determine the effects of charging on the energy levels and on the SDHD. In the first place the lowest hole states need to be well bound for all reasonable densities, yielding a minimum well width of about $30 \AA$. Second, the lowest $k=0$ solutions are to be mainly localized in one of the wells, but need to have amplitude in both wells. This means that the width of the central barrier should be of the order of a few times the penetration length of the heavy-hole wave function $(\approx 25-60$ $\AA$ ). Furthermore, one wants to avoid the complexity and ambiguity of many, hardly separated, bound states, occurring in wide wells $(>100 \AA)$. Within these limits, every ADQW behaves qualitatively the same, which allows us to consider one typical structure as general. The structure under consideration in this section consists of a $59.4-\AA$ (21 ML of GaAs) and a $42.4-\AA$ (15-ML) well, separated by a $33.9-\AA$ (12-ML) barrier, with an $\mathrm{Al}$ content of $25 \%$. In order to enhance the confinement of the bound states, the outer barriers have an $\mathrm{Al}$ content of $40 \%$. The spacer layer between the doping and the nearest $\mathrm{GaAs} / \mathrm{Al}_{x} \mathrm{Ga}_{1-x} \mathrm{As}$ interface is taken to be $33.9 \AA$. The thickness of the confining barriers is chosen such that no significant change of observables occurs upon a further increase.

\section{A. Zero in-plane wave vector}

In Fig. 1 the wave functions of the first two light-and heavy-hole levels are depicted, together with the first two electron levels, for a sheet density of $1.5 \times 10^{16} \mathrm{~m}^{-2}$. As expected, the ground states of the heavy, light, and electron levels are predominantly localized in the wide well and have an even character with respect to the middle of the central barrier, whereas the first excited states are odd-character functions, localized mainly in the narrow well. More remarkable is the density dependence of the energy separation between the first and second heavy-hole levels (HHO and

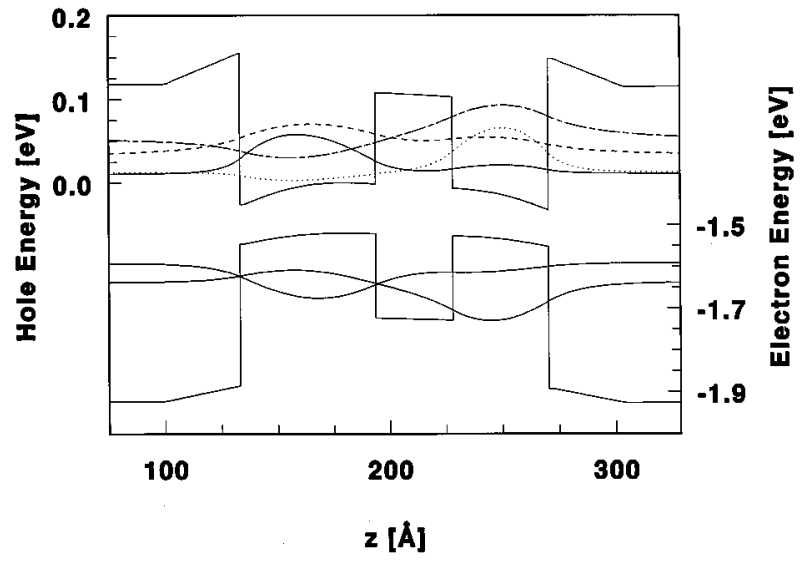

FIG. 1. Energy positions and envelope functions of the first hole and electron levels in a self-consistent potential at zero in-plane wave vector. The hole density is $1.5 \times 10^{16} \mathrm{~m}^{-2}$. Hole levels: solid line, HH0; dotted line, HH1; dashed line, LH0; dash-dotted line, LH1. Electron levels, top to bottom: $E 0$ and $E 1$.

HH1); see Fig. 2. Upon the first introduction of charge, the HH1 level rapidly shifts down to a few meV above the HHO level at $p \approx 0.2 \times 10^{16} \mathrm{~m}^{-2}$. For higher sheet concentrations the $\mathrm{HH} 0-\mathrm{HH} 1$ separation remains independent of charge density. This behavior is a direct consequence of the electrostatic effect of the space charge.

For very low carrier concentrations, all charge will condense in the lowest level, HH0. Since HHO is mainly localized in the wide well, the narrow well will be shifted down with respect to the wide well by the resulting electrostatic potential. Consequently, the HH1 level, having its maximum amplitude in the narrow well, shifts down towards the HHO level. Figure 1 illustrates this. A crossing of the HHO and HH1 levels is avoided by the fact that the density of states (DOS) is almost the same for both levels: as soon as HH1 drops below the Fermi level, all extra charge is equally distributed over both wells, resulting in a status quo for the relative positions of $\mathrm{HHO}$ and $\mathrm{HH} 1$. This point is indicated by the arrow in Fig. 2 .

The density at which the HHO and HH1 levels would become degenerate can be obtained by a simple back-of-the-

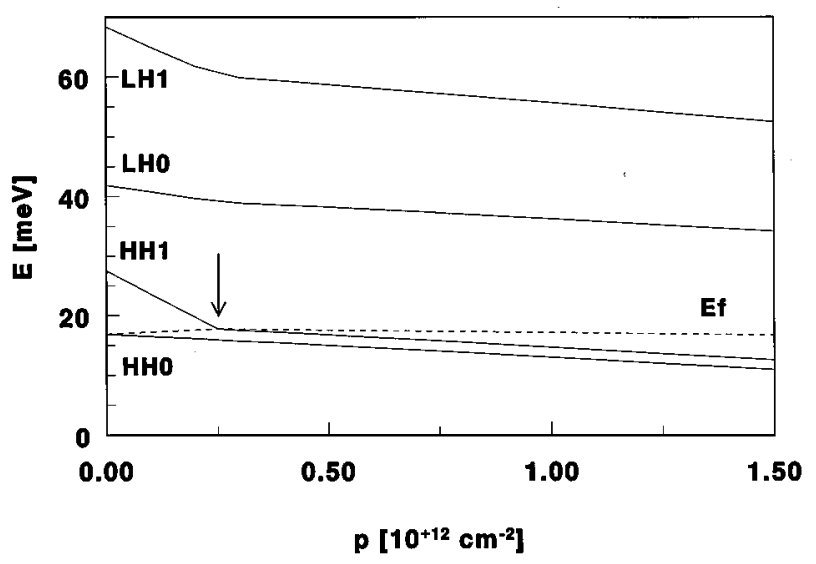

FIG. 2. Energies of LH1, LH0, HH1, and HH0 levels and Fermi energy as a function of doping concentration for the standard structure (see Fig. 1). 

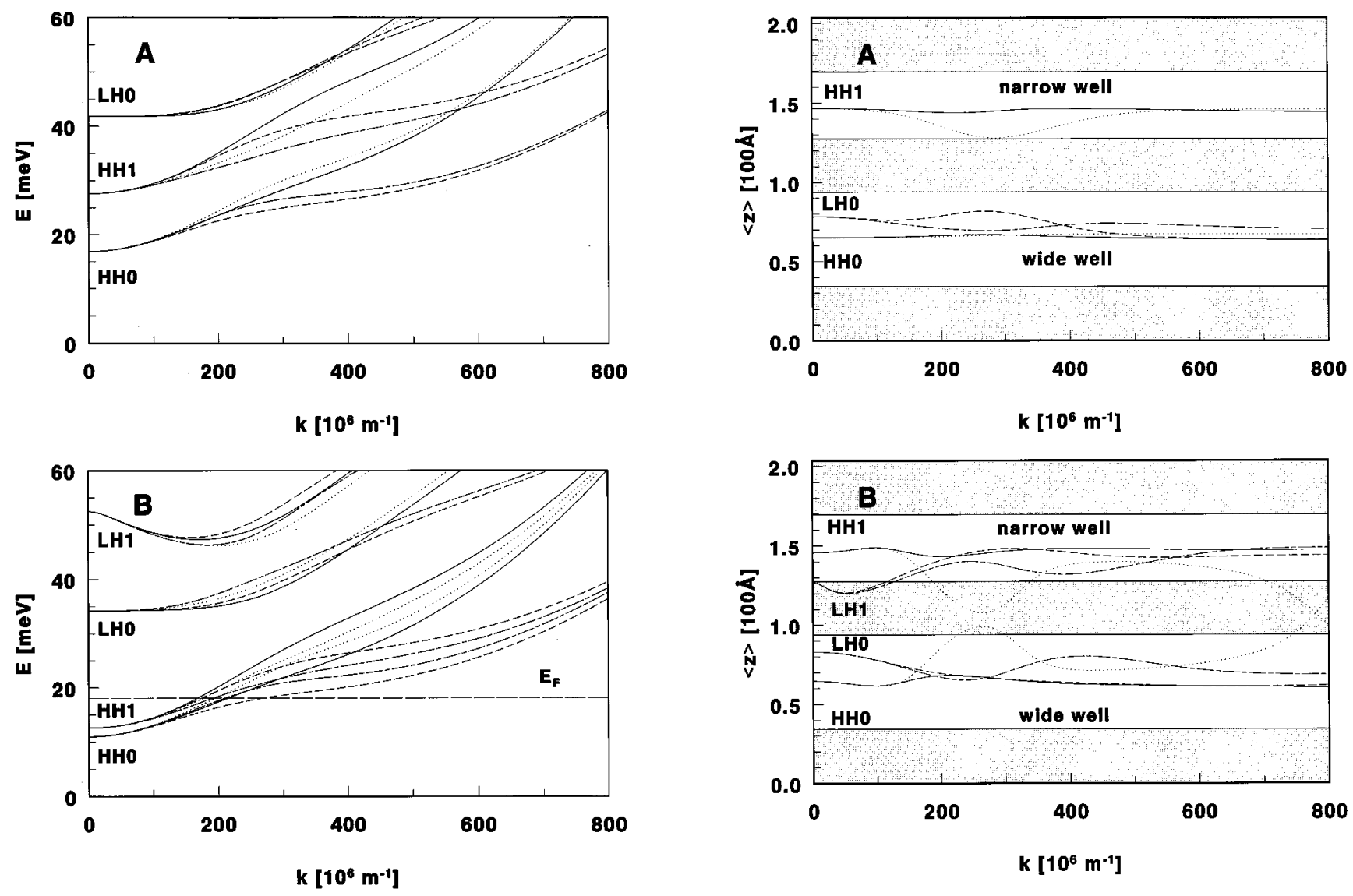

FIG. 3. (a) Dispersion relation of an empty 59.4-33.9-42.4 ̊̊ ADWQ. (b) Same as (a), but for a density of $1.5 \times 10^{16} \mathrm{~m}^{-2}$. Solid lines, spin up, $\langle 10\rangle$ direction; dotted lines, spin down, $\langle 10\rangle$; dashed lines, spin up, $\langle 11\rangle$; dash-dotted lines, spin down, $\langle 11\rangle$.

envelope calculation of the electrostatic potential of a single charged plate $V=p e d / 2 \epsilon_{0} \epsilon_{r}$. Taking $d$ as the distance between the centers of both wells and $V$ the potential difference between $\mathrm{HHO}$ and $\mathrm{HH} 1$ in the $p=0$ situation, we find $p=0.16 \times 10^{16} \mathrm{~m}^{-2}$, in reasonable agreement with the exact result. It is worthwhile to note that the above-described alignment effect is not restricted to hole gasses, nor to the specific dimensioning of the ADQW.

\section{B. Nonzero in-plane wave vector}

The effect of self-consistency on the dispersion relations of an ADQW is depicted in Fig. 3. Clearly visible is the downward shift of the first excited heavy- and light-hole bands in the degenerate system [Fig. 3(b)], compared to the empty system [Fig. 3(a)], due to the charge-induced band bending. Note also the very similar dispersion curves for $\mathrm{HH} 0$ and $\mathrm{HH} 1$, implying the equality of their DOS. The spindependent hole delocalization for the empty and filled structures is represented in Fig. 4. Plotted is the expectation value of the $z$ operator, $\langle z\rangle=\int \Psi^{*} z \Psi d z$ with $\Psi$ the corresponding envelope function, for both spin directions of HHO, LHO, and HH1 versus $|k|$ in the $\langle 10\rangle$ direction. The zero of the $z$ axis is chosen at the left $\delta$-doping layer. Note that the presence of charge does not affect $\langle z\rangle$ for $\mathrm{HH} 0$ and $\mathrm{HH} 1$ at $k=0$, but does so for the LHO. This is a result of the smaller penetration length of the heavy-bound states, effectively localiz-

FIG. 4. (a) $\langle z\rangle$ versus $|k|$ for the ADQW of Fig. 3(a) in the $\langle 10\rangle$ direction. (b) Same as (a), but for Fig. 3(b). Solid lines, HH, spin up; dotted lines, HH, spin down; dashed lines, LH, spin up; dashdotted lines, LH, spin down. The shaded areas indicate the $\mathrm{Al}_{x} \mathrm{Ga}_{1-x} \mathrm{As}$ barriers.

ing $\mathrm{HH} 0$ and $\mathrm{HH} 1$ almost completely in the separate wells, making $\langle z\rangle$ independent of their relative energy positions. The results for the empty ADQW confirm the observation by Goldoni and Fasolino ${ }^{6}$ that the HHO state, in contrast to the HH1 and LH0 states, does not show any appreciable spindependent tunneling, i.e., the spin-up and spin-down states are not macroscopically separated. However, the spatial separation of spin-up and -down states at $k \neq 0$ is clearly enhanced for the filled system, resulting in almost equal splittings for $\mathrm{HH} 0$ and $\mathrm{HH} 1$.

In Fig. 5 the light and heavy components of the envelope functions are shown at maximum spin splitting $\left(k=2.6 \times 10^{8}\right.$ $\mathrm{m}^{-1}$ ). Only the components that are shifted towards the barrier obtain significant amplitude in the opposite well and will therefore "feel" the potential asymmetry and become delocalized. Quite remarkable is the change of parity of the HHO and $\mathrm{HH} 1$ wave functions with respect to the $k=0$ case: $\mathrm{HH} 0$ has one node and $\mathrm{HH} 1$ none. This is due to the fact that the $\mathrm{HH} 0$ and $\mathrm{HH} 1$ have already anticrossed at the present wave number [Fig. 3(b)] resulting in an exchange of character.

The spin-dependent hole delocalization of the filled $\mathrm{ADQW}$ is augmented with respect to the empty system by two effects: first, by the extra asymmetry resulting from the band bending and second, and most important, by the stronger mixing between the $\mathrm{HH} 0$ and $\mathrm{HH} 1$ bands at finite $k$, due to their small energy separation. To strengthen our argument 


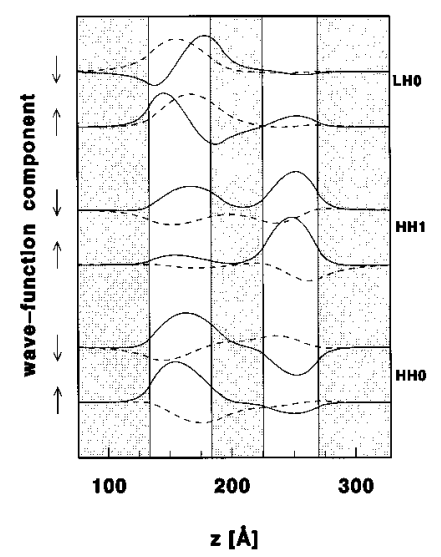

FIG. 5. Envelope function of wave-function components at $k=2.6 \times 10^{8} \mathrm{~m}^{-1}$ in the $\langle 10\rangle$ direction for the $59.4-33.9-42.4 \AA$ ADQW with a density of $1.5 \times 10^{16} \mathrm{~m}^{-2}$. Solid lines, HH component; dashed lines, LH component.

about the general character of the SDHD enhancement and to prove that this enhancement is mainly due to an increase of the coupling between $\mathrm{HHO}$ and $\mathrm{HH} 1$ levels, we calculated $\langle z\rangle(k)$ traces for different densities. The results are depicted in Fig. 6. The main graph shows $\langle z\rangle(k)$ traces for an empty well, for $p=0.20 \times 10^{16} \mathrm{~m}^{-2}$ (just before HH1 becomes populated) and for $p=0.30 \times 10^{12} \mathrm{~cm}^{-2}$ (just after HH1 is populated). Note the strong increase of spin splitting of the $\mathrm{HH} 0$ state at the density the $\mathrm{HH} 1$ band becomes populated. This is also visible in the inset, where the maximum "macroscopic" splitting $\left(\left|\langle z\rangle_{\text {up }}-\langle z\rangle_{\text {down }}\right|\right)$ between spin-up and spin-down branches is shown for both HHO and HH1. It is important to note the stability of the spin splitting for densities above $p=0.30 \times 10^{12} \mathrm{~cm}^{-2}$, showing the relative unimportance of the increasing asymmetry of the structure.

Again it should be stressed that the enhancement of the SDHD by charging effects is not restricted to the particular structure discussed above. The reason is that both responsible mechanisms, increasing asymmetry due to band bending and stronger mixing of $\mathrm{HH} 0$ and $\mathrm{HH} 1$ states due to their decreased separation in energy, are inherent to any ADQW,

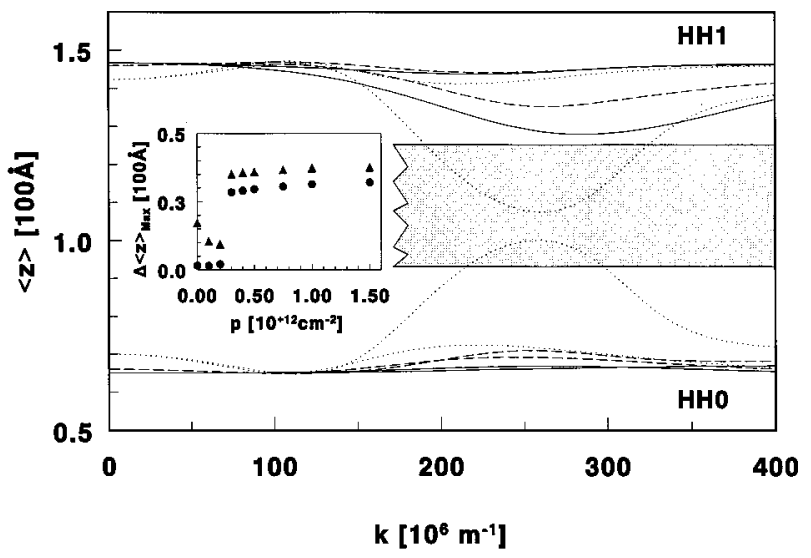

FIG. 6. $\langle z\rangle$ versus $|k|$ for the 59.4-33.9-42.4 $\AA$ ADQW for $p=0$ (solid lines), $p=0.20 \times 10^{16} \mathrm{~m}^{-2}$ (dashed lines), and $p=0.30 \times 10^{16} \mathrm{~m}^{-2}$ (dotted lines). The shaded area indicates the central $\mathrm{Al}_{x} \mathrm{Ga}_{1-x} \mathrm{As}$ barrier. Inset: maximum macroscopic spinsplitting versus density. Circles, HH0; triangles, HH1.

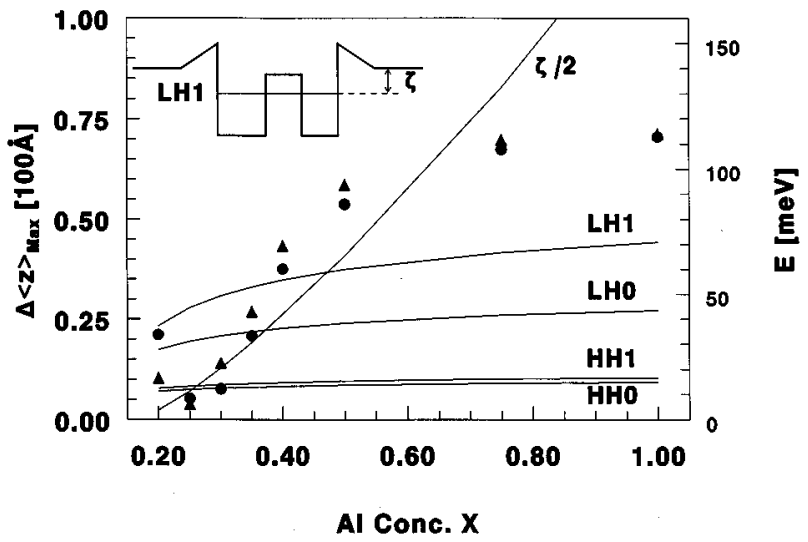

FIG. 7. Left axis: maximum macroscopic spin splitting versus $\mathrm{Al}$ concentration in the confining barriers of the standard structure. Circles, HH0; triangles, HH1. Right axis: energies of the lowest light and heavy states, together with the LH1 "effective bonding energy," $\zeta$.

as shown in Sec. III A. However, the exact behavior of any structure is a function of its particular dispersion relation and wave functions and is therefore dependent on sample dimensions.

Two more notes have to be made on the enhancement of spin-dependent hole delocalization by charging effects. First, the SDHD is, for both systems, induced by different couplings with other hole levels for spin-up and -down states at nonzero wave vector ${ }^{6}$ and does not require resonance of levels. Second, the HH0-HH1 hybridization is not a result of a direct coupling, since (1) does not contain direct coupling terms between states with the same $\left|m_{J}\right|$. Consequently, the HH0-HH1 mixing must take place through interaction with light-hole bands. It must be stated that the SDHD of the HHO and $\mathrm{HH} 1$ wave functions cannot arise from a direct hybridization with LHO and LH1 wave functions, respectively, since this would result in a shift away from the central barrier at $k=2.6 \times 10^{8} \mathrm{~m}^{-1}$ [Fig. 4(b)].

The importance of well-bound LH states for the enhancement of SDHD is illustrated in Fig. 7, where the maximum spin splitting of $\mathrm{HH} 0$ and $\mathrm{HH} 1$ is depicted as a function of $\mathrm{Al}$ concentration in the confining barriers. The $\mathrm{Al}$ content of the central barrier is kept at $25 \%$. Increasing this barrier only reduces the SDHD, since it weakens the coupling between the two wells. The SDHD is strongly enhanced when the Al concentration in the confining barriers is more than $30 \%$. This coincides with a significant increase of the localization of the lowest light-hole states (right $y$ axis), whereas the localization of the lowest heavy-hole states is not significantly modified for $x>20 \%$. Both the maximum spin splitting and the confinement of the LH states, as deduced from the flattening of the $\mathrm{LH}$ energy curves, become saturated for $x>50 \%$. It is interesting to note that, although the maximum spin splitting is strongly modified by the Al concentration $x$, the wave number at which this occurs does not significantly shift with $x$.

So far, it has been shown that the spin-dependent delocalization of hole levels is strongly dependent on the interactions between heavy- and light-hole bands. Due to the warped nature of the valence bands, also a direction dependence of SDHD is to be expected. Since the extrema in the 


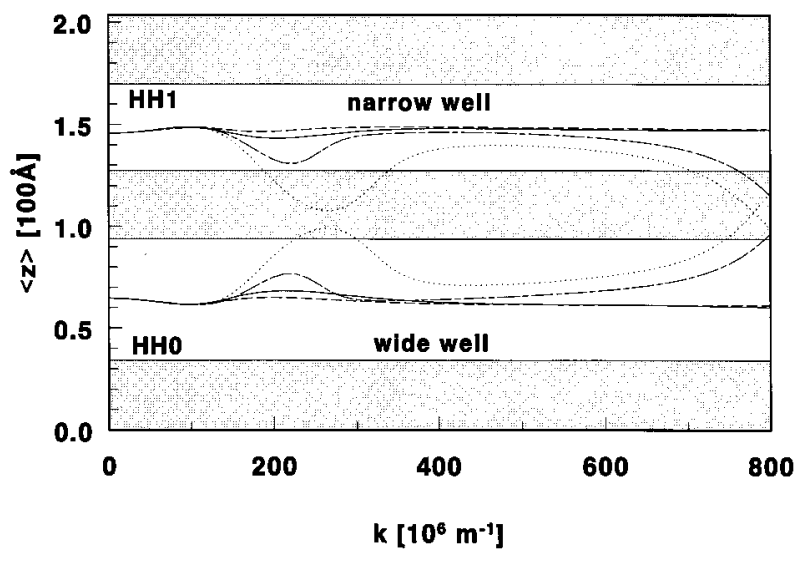

FIG. 8. $\langle z\rangle$ versus $|k|$ for the ADQW of Fig. 3(b) in the $\langle 10\rangle$ and $\langle 11\rangle$ directions. Solid lines, spin up, $\langle 10\rangle$; dotted lines, spin down, $\langle 10\rangle$; dashed lines, spin up, $\langle 11\rangle$; dash-dotted lines, spin down, $\langle 11\rangle$.

dispersion surface due to warping occur along the $\langle 10\rangle$ and $\langle 11\rangle$ directions, we also calculated $\langle z\rangle$ in these directions; see Fig. 8. The spin-dependent delocalization is clearly the most pronounced in the $\langle 10\rangle$ direction, which confirms our argument that the spatial separation of HH spin-up and -down states arises from interactions with LH bands. The dispersion relations of $\mathrm{HH} 0$ and $\mathrm{HH} 1$ in both directions are rather similar [Fig. 3(b)], but the $\langle 11\rangle$ direction shows far less anticrossing behavior between $\mathrm{HH}$ and $\mathrm{LH}$ than the $\langle 10\rangle$ direction. This results from a weaker interaction between light and heavy bands in the $\langle 11\rangle$ direction (see also the second remark in the preceding paragraph).

\section{EXPERIMENT}

In order to check our calculations, the sample described in Sec. III was grown with $25 \% \mathrm{Al}$ barriers. The doping concentration was chosen such that the Fermi vector would lay approximately at maximum spin splitting, i.e., $k_{F}=2.3 \times 10^{8}$ $\mathrm{m}^{-1}$ [Fig. 4(b)]. The aimed sheet density was $1.5 \times 10^{16} \mathrm{~m}^{-2}$.

\section{A. Transport experiments}

The "alignment" effect described in Sec. III A is confirmed by Shubnikov-de Haas measurements on the aforementioned structure; see Fig. 9. A plot of $1 / B$ versus the extremum number (see the inset) revealed three distinct periodicities, with slopes related as 1:2:4. This is interpreted as follows: In the high-field limit the Landau levels are nondegenerate, so each $\rho_{x x}$ minimum corresponds to a filling-factor change of unity. In other words, Landau levels pass through the Fermi level one by one. Therefore the low- and intermediate-field regimes must correspond to the situations where four and two Landau levels pass through the Fermi level at the same time, respectively, and the filling factor changes in steps of four and two at each $\rho_{x x}$ minimum. The values and positions of the Hall plateaus give further evidence for this view. From both Shubnikov-de Haas and Hall measurements we found a sheet density of $1.5 \times 10^{16} \mathrm{~m}^{-2}$. From Fig. 2 it becomes clear that a Landau level broadening of about $1 \mathrm{meV}$, which is quite reasonable, will prevent the HHO and HH1 Landau levels from being resolved in the low-field regime. Since spin-up and -down states are also

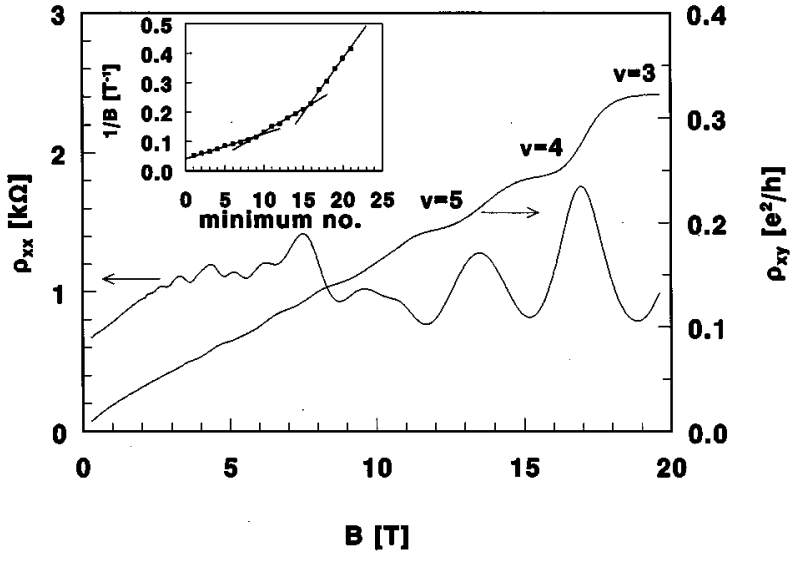

FIG. 9. Shubnikov-de Haas and Hall measurement on the standard ADQW with a carrier density of $1.5 \times 10^{16} \mathrm{~m}^{-2}$. Insert: $1 / B$ of the $\rho_{x x}$ minima versus order number (not filling factor) of the minima. Note the three distinct periodicities, related as 1:2:4.

degenerate at low fields, Landau levels will appear to be fourfold degenerate. Upon increasing the magnetic field, both the HHO-HH1 "degeneracy" and the spin degeneracy are lifted. Since this will most likely occur at different field strengths the described Shubnikov-de Haas behavior will result.

\section{B. Optical experiments}

The necessity of self-consistency in degenerate systems is shown by the simulation of photoluminescence excitation (PLE) spectra of coupled quantum wells. The top panel of Fig. 10 displays such a spectrum of the same structure as the one on which the Shubnikov-de Haas measurements were performed, accompanied by the photoluminescence (PL) lines. The PL and PLE experiments were performed at $4.2 \mathrm{~K}$, using normal incident, unpolarized light from a Ti:sapphire laser.

When we only take direct optical transitions into account, the PLE intensity is proportional to the product of the square of the matrix element and the joint density of states, integrated over the full $k$ space:

$$
I(E)=\int d k M(k)^{2} \delta\left(E_{t}(k)-E\right)=\oint_{E} d k \frac{M(k)^{2}}{\nabla E_{t}(k)},
$$

where the matrix element $M(k)$ is given by

$$
\begin{gathered}
M(k)=\left\langle\Psi_{\text {electron }}|p \epsilon| \Psi_{\text {hole }}\right\rangle \quad \text { if } E_{h}>E_{F}, \\
M(k)=0 \quad \text { if } E_{h} \leqslant E_{F} .
\end{gathered}
$$

Here $E_{t}=E_{h}+E_{e}+E_{\text {gap }}$, with $E_{h}\left(E_{e}\right)$ the energy of the involved hole (electron) level, with respect to the top (bottom) of the valence (conduction) band, $E_{\text {gap }}$ is the band gap of GaAs, and $E_{F}$ is the Fermi energy. The Fermi energy was obtained by integration over the calculated dispersion surface. The Stokes shift is accounted for by $M(k)$, i.e., only empty states (at $T=0)$ contribute to the PLE spectrum.

The dispersion relations and matrix elements were calculated for both interface conditions (flux or wave-function continuity) in either a self-consistent or a non-self-consistent 


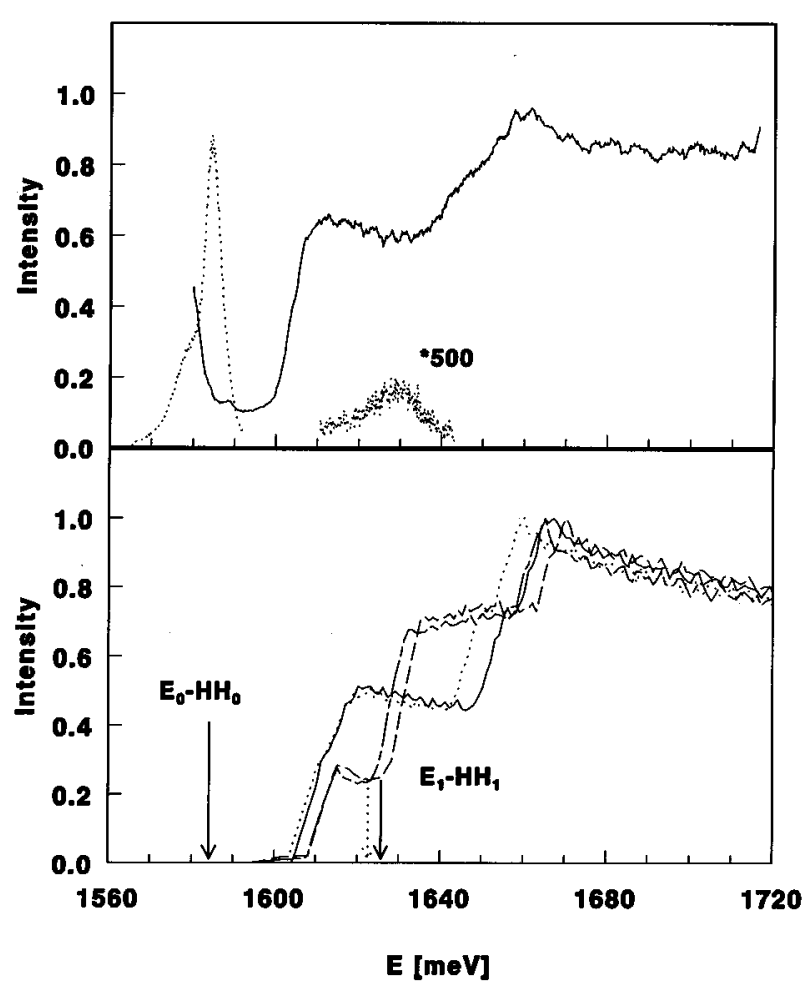

FIG. 10. Top panel: experimental PL (dotted lines) and PLE (solid lines) spectra at $4.2 \mathrm{~K}$ of the 59.4-33.9-42.4 $\AA$ ADWQ with a density of $1.5 \times 10^{16} \mathrm{~m}^{-2}$ and $25 \% \mathrm{Al}$ barriers. Bottom panel: calculated PLE spectra of the same structure. The arrows denote the calculated PL onsets. Solid line, self-consistent, wave-functioncontinuity interface conditions (see the text for details); dotted line, self-consistent, flux-conserving IC's; dashed lines, not-selfconsistent, wave-function-continuity IC's; dash-dotted line, nonself-consistent, flux-conserving IC's.

calculation, yielding four different sets of $M(k)$ and $E_{t}(k)$. The resulting $I(E)$ are depicted in the lower panel of Fig. 10. The only fitting parameter is a rigid shift in the band edge commonly encountered in doped systems and known as band-gap renormalization, which is used to position the first PL peak, indicated by the arrow. The applied values are around $7 \mathrm{meV}$ for the "flux-conserving" calculations and around $11.5 \mathrm{meV}$ for the "continuous-wave-function" calculations, which seems reasonable. ${ }^{26}$ Clearly visible in Fig. 10, there is excellent agreement between the experimental curve and the self-consistent simulations, whereas the non-selfconsistent simulations deviate significantly from the experimental curve. The choice of interface conditions seems a rather arbitrary one for these calculations and it would be unwise to draw any conclusions from the minor deviations present. We will focus on the continuous-wave-function simulation in our last remark concerning Fig. 10.

A more detailed comparison of self-consistent and nonself-consistent calculated traces shows the need of calculating the exact matrix elements in order to obtain the correct intensities in the PLE spectrum. For example, for both calculations the onsets of the first PLE step ( $E 0$ to $\mathrm{HHO}$ at $\sim 1.59 \mathrm{eV})$ and of the second step (E1 to HH1 at $\sim 1.63 \mathrm{eV})$ lay on approximately the right energy positions, when the Moss-Burstein shift is properly taken into account. The significant differences between both calculations around these points arise mainly as a result of different matrix elements since the DOS of each band are almost independent upon the inclusion of the effects of the charge distribution.

\section{SUMMARY}

We have presented an exact and self-consistent method for solving the coupled Poisson equation and the $4 \times 4$ Luttinger Hamiltonian, with inclusion of anisotropy. The need for the inclusion of Coulombic effects on an asymmetric double quantum well has been investigated and shown to be of great importance for a meaningful comparison with transport experiments, as well as with optical experiments. In the same structure, the spin-dependent hole delocalization becomes strongly enhanced by the self-consistency due to a decrease of the energy separation between the first and second heavy-hole subbands and an increase in the asymmetry of the confining potential. The importance of confined lighthole states for the SDHD was shown by variation of the height of the confining barriers. Furthermore, the influence of the interface conditions on the dispersion relations and wave function was inspected and found to be of minor importance for the structures under consideration.

Note added. During the preparation of this manuscript we received a copy of work, prior to publication, by Goldoni and Fasolino ${ }^{24}$ in which they present a very similar method to solve the Luttinger Hamiltonian exactly for one $k$ dimension. Both methods are flexible in nature and yield direct access to wave functions.

\section{ACKNOWLEDGMENTS}

We would like to acknowledge Andrei Silov and Steven Theeuwen for valuable discussions and important contributions to the described computer software during the early stages of this work.
${ }^{1}$ See, e.g., E. P. O'Reilly, Semicond. Sci. Technol. 4, 121 (1989), and references therein.

${ }^{2}$ M. Z. Tidrow, K. K. Choi, C. Y. Lee, W. H. Chang, F. J. Towner, and J. S. Ahearn, Appl. Phys. Lett. 64, 1268 (1994).

${ }^{3}$ K. Bernhard, A. Zrenner, G. Bohm, G. Trankle, and G. Weiman, Solid State Electron. 37, 1307 (1994).

${ }^{4}$ Y. Ohno, M. Tsuchiya, and H. Sakaki, Appl. Phys. Lett. 62, 1952 (1993).

${ }^{5}$ O. E. Raichev and F. T. Vasko, Phys. Rev. B 51, 7116 (1995).
${ }^{6}$ G. Goldoni and A. Fasolino, Phys. Rev. Lett. 69, 2567 (1992).

${ }^{7}$ G. Goldoni and A. Fasolino, Surf. Sci. 305, 333 (1994).

${ }^{8}$ P. Lefebvre, P. Bonnel, B. Gil, and H. Mathieu, Phys. Rev. B 44, 5635 (1991).

${ }^{9}$ V. Pereyra, in Lecture Notes in Computer Science, edited by B. Childs, M. Scott, J. W. Daniel, E. Denman, and P. Nelson (Springer, Berlin, 1979), Vol. 76.

${ }^{10}$ T. Ando, J. Phys. Soc. Jpn. 54, 1528 (1985). 
${ }^{11}$ D. A. Broido and L. J. Sham, Phys. Rev. B 31, 888 (1985).

${ }^{12}$ U. Ekenberg and M. Altarelli, Phys. Rev. B 32, 3712 (1985).

${ }^{13}$ S.-R. Eric Yang, D. A. Broido, and L. J. Sham, Phys. Rev. B 32, 6630 (1985).

${ }^{14}$ E. Bangert and G. Landwehr, Surf. Sci. 170, 593 (1986).

${ }^{15}$ For a general discussion of existing approaches see, e.g., R. Winkler and U. Rössler, Phys. Rev. B 48, 8918 (1993).

${ }^{16}$ L. C. Andreani, A. Pasquarello, and F. Bassani, Phys. Rev. B 36, 5887 (1987).

${ }^{17}$ G. Edwards, E. C. Valdares, and F. W. Sheard, Phys. Rev. B 50, 8493 (1994).

${ }^{18}$ M. Kemerink, P. M. Koenraad, R. v. Schayk, P. C. M. Christianen, J. C. Maan, and J. H. Wolter (unpublished).

${ }^{19}$ J. M. Luttinger, Phys. Rev. 102, 1030 (1956).

${ }^{20}$ G. Bastard, Wave Mechanics Applied to Semiconductor Heterostructures (Les Editions de Physique, Les Ulis, 1988).

${ }^{21}$ S. L. Chuang, Phys. Rev. B 40, 10379 (1989).

${ }^{22}$ It is still a point of discussion which interface conditions should be applied in the effective-mass approximation. From a physical point of view, flux has to be conserved at any interface. However, to define the ratio of the wave-function derivatives as the ratio of the effective masses, the Bloch basis functions need to be the same in GaAs and AlAs. Although this is a crucial assumption in the effective-mass approximation, it is not fully true. See J. P. Cuypers and W. van Haeringen, Phys. Rev. B 47, 10310 (1993).

${ }^{23}$ For a review of problems and solutions concerning interface conditions in effective-mass theory see M. G. Burt, J. Phys. Condens. Matter 4, 6651 (1992).

${ }^{24}$ G. Goldoni and A. Fasolino, Phys. Rev. B 51, 9903 (1995).

${ }^{25}$ Physics of Group IV Elements and III-V Compounds, edited by O. Madelung, M. Schultz, and H. Weiss, Landolt-Börnstein, New Series, Group III, Vol. 17, Pt. a (Springer-Verlag, Berlin, 1982); ibid., Vol. 22, Pt. a (Springer-Verlag, Berlin, 1987).

${ }^{26}$ S. Haacke, R. Zimmermann, D. Bimberg, H. Kal, D. E. Mars, and J. N. Miller, Phys. Rev. B 45, 1736 (1992). 